Article

\title{
Two-Stage Prediction of the Effects of Imidazolium and Pyridinium Ionic Liquid Mixtures on Luciferase
}

\author{
Hui-Lin Ge ${ }^{1,2, *}$, Shu-Shen Liu ${ }^{2, *}$, Bing-Xia Su ${ }^{1}$ and Xiang-Wei Zhu ${ }^{3}$
}

1 Hainan Provincial Key Laboratory of Quality and Safety for Tropical Fruits and Vegetables, Analysis and Testing Center, Chinese Academy of Tropical Agricultural Sciences, Haikou 571101, China; E-Mail: sublin22@163.com

2 Key Laboratory of Yangtze Aquatic Environment, Ministry of Education, College of Environmental Science and Engineering, Tongji University, Shanghai 200092, China

3 College of Resource and Environment, Qingdao Agricultural University, Qingdao 266109, China; E-Mail: shinevip@163.com

* Authors to whom correspondence should be addressed; E-Mails: huilinge@126.com (H.-L.G.); ssliuhl@263.net (S.-S.L.); Tel./Fax: +86-898-6689-5011 (H.-L.G.).

Received: 20 March 2014; in revised form: 15 May 2014 / Accepted: 19 May 2014 /

Published: 23 May 2014

\begin{abstract}
The predicted toxicity of mixtures of imidazolium and pyridinium ionic liquids (ILs) in the ratios of their $\mathrm{EC}_{50}, \mathrm{EC}_{10}$, and NOEC (no observed effect concentration) were compared to the observed toxicity of these mixtures on luciferase. The toxicities of $\mathrm{EC}_{50}$ ratio mixture can be effectively predicted by two-stage prediction (TSP) method, but were overestimated by the concentration addition (CA) model and underestimated by the independent action (IA) model. The toxicities of $\mathrm{EC}_{10}$ ratio mixtures can be basically predicted by TSP and CA, but were underestimated by IA. The toxicities of NOEC ratio mixtures can be predicted by TSP and CA in a certain concentration range, but were underestimated by IA. Our results support the use of TSP as a default approach for predicting the combined effect of different types of ILs at the molecular level. In addition, mixtures of ILs mixed at NOEC and $\mathrm{EC}_{10}$ could cause significant effects of $64.1 \%$ and $97.7 \%$, respectively. Therefore, we should pay high attention to the combined effects in mixture risk assessment.
\end{abstract}

Keywords: ionic liquids; luciferase; molecular level; joint toxicity; concentration addition; independent action; two-stage prediction; NOEC; mixture risk assessment 


\section{Introduction}

Organisms are usually exposed to pollutant mixtures in the environment [1]. The assessment and prediction of mixture effects is generally based on two additive reference models: concentration addition (CA) and independent action (IA). CA is generally applied in predicting the mixture toxicity of similarly acting chemicals [2], while IA is generally appropriate for the mixture of dissimilarly acting chemicals [3]. Later, Junghans proposed a two-stage prediction (TSP) method that combined CA and IA in a stepwise manner [4]. TSP has been used to predict mixture toxicities of different types of chemicals [5,6].

Ionic liquids (ILs) are novel organic salts with low melting points that have enormous potential for industrial use as green replacements for harmful volatile organic solvents [7]. The application areas of ILs include catalysis, extraction, synthesis, dissolution, nuclear industry, and food science [8]. Although ILs will not cause air pollution because of their negligible vapor pressures, some of them still present a non-negligible solubility in water, thus leading to aquatic environmental risks [9]. Several studies have reported the biological effects of single ILs on the basis of different toxicological test systems such as enzymes (e.g., acetylcholinesterase), bacteria (e.g., Vibrio fischeri), algae (e.g., Selenastrum capricornutum), mammalian cells (e.g., MCF-7 cell), plants (e.g., wheat and cress), invertebrates (e.g., zebra mussel), and vertebrates (e.g., Danio rerio) [10-16].

Mixtures of ionic liquids are increasingly applied in practical applications such as solvents for chemical synthesis and process chemistry, electrochemistry, chromatography and heat transfer fluids [17]. It is of great importance to predict and assess their combined effects before any likely industrial release into the environment. Several studies have reported the combined effects of ILs. For example, the effects of imidazolium IL mixtures on Triticum aestivum and Scenedesmus vacuolatus were underestimated by the CA and IA models [18]. Imidazolium ILs can cause multiple toxic interactions (addition, synergism, and antagonism) in different composition and concentration ranges [19]. The synergism and antagonism of imidazolium ILs are well correlated to the concentration ratio of ILs with $\mathrm{BF}_{4}^{-}$[20]. Nevertheless, little is known about the combined toxicity of different types of ILs, including imidazolium and pyridimium ILs. Important questions are: (i) can their mixture toxicities be predicted by CA, IA or TSP; (ii) what is the type of their toxic interaction (addition, synergism or antagonism); and (iii) what is the location relationship among the concentration-response curves (CRCs) predicted by CA, IA, and TSP for their mixtures? Although some of these issues have been preliminarily investigated in single types of ILs such as imidazolium ILs, it is necessary to further explore and validate this for mixtures of different types of ILs.

Luciferase luminescence is a process in which the luciferase catalyzes the oxidation of the substrate D-luciferin and the energy transfer from ATP to D-luciferin to yield light. Because the luciferase luminescence can be affected by chemicals, it has been applied as a test system to characterize the toxicities of single chemicals [21] and mixtures [22]. Based on the luciferase toxicity test, we demonstrated that the effects of mixtures of ten ILs with J-shaped CRC can be predicted by the CA model [23]. In the present study, we evaluate the effects of mixtures of imidazolium and pyridimium ILs with S-shaped CRC. Mixture effects of ILs in the ratios of their $\mathrm{EC}_{50}, \mathrm{EC}_{10}$, and NOEC on luciferase were tested based on microplate toxicity analysis. Combined effects were evaluated by comparing the predicted effects by CA, IA, and TSP to the observed effects of these mixtures. 


\section{Results and Discussion}

\subsection{Single Toxicity}

The regression models and the estimated parameters of the toxicity of single ILs on luciferase are summarized in Table 1 and the resulting CRCs are visualized in Figure 1. These CRCs can be effectively described by the Logit or Weibull model with RMSE $(<0.06)$ and $R^{2}(>0.95)$, indicating good relationships between the exposure concentrations of ILs and the inhibition effects. The variability of the blank control in our test was controlled within $\pm 10 \%$. All $\mathrm{EC}_{50}, \mathrm{EC}_{10}$ and the NOEC values are shown in Table 1. According to their $\mathrm{EC}_{50}$ s, the toxicity order of single ILs was IL4 > IL6 $>$ IL5 > IL10 > IL8 > IL7 > IL1 > IL11 > IL9 > IL3 > IL2 (for the meaning of the abbreviations see below). Both the present study and a previous study [23] all indicated that the ILs with $\mathrm{BF}_{4}{ }^{-}$anions showed higher luciferase toxicity. Moreover, ILs with $\mathrm{BF}_{4}{ }^{-}$anions were also inclined to produce higher toxicity on other organisms such as the luminescent bacterium Q67 [24] and MCF-7 mammalian cells [13]. The high toxicity of ILs with fluoride-containing anions could be due to the hydrolytic cleavage resulting in the formation of free fluoride ions [25]. Egorova and Ananikov have reviewed the main factors modulating the toxicity of ILs: (i) alkyl chain length and side chain functionalization in the cation; (ii) nature of the anion and cation; (iii) mutual influence of the anion and cation [26].

By inserting the NOEC values (Table 1) into the concentration-response functions $(F)$, it can be calculated that they correspond to effects ranging from $0.1 \%$ for IL5 to $11.3 \%$ for IL3 with an arithmetic mean of 3\%. The NOEC was obtained based on conventional hypothesis testing for each tested concentration. Many ecotoxicologists are aware of the shortcomings of the NOEC. The most often cited concerns include: the NOEC is constrained to be one of the test concentrations; confidence intervals cannot be calculated for NOEC; NOEC cannot always be determined; NOEC is controlled by the concentration interval, the data variability, the number of replicates, and the selected significance level [27]. On the other hand, the $\mathrm{EC}_{x}$ (the concentration causing an effect of $x$ percent) was estimated from a concentration-response model based on statistical regression analysis of whole data sets, so NOECs are innately more variable than $\mathrm{EC}_{x}$ point estimates. Low effect $\mathrm{EC}_{x}$ values have been suggested as appropriate alternatives to NOEC [28]. Using $\mathrm{EC}_{x}$ to replace NOEC requires the value of $x$ to be specified. Van der Hoeven et al. suggested that the preferred value of $x$ would be 5 or 10 percent [29]. In fact, the confidence interval for low effect $\mathrm{EC}_{x}$ may be very wide and sometimes even missing such as the $\mathrm{EC}_{10}$ of IL3 and IL7 in our study, so $\mathrm{EC}_{10}$ may be a more appropriate choice to replace NOEC. 
Table 1. Concentration-response models of individual ionic liquids and their mixtures on the inhibitory effects of luciferase luminescence and some statistics.

\begin{tabular}{|c|c|c|c|c|c|c|c|c|c|c|c|c|c|}
\hline No. & $\boldsymbol{F}$ & $\alpha$ & $\beta$ & $R^{2}$ & RMSE & $f$ & $\mathrm{EC}_{50}$ & $\mathrm{EC}_{10}$ & NOEC & $t$ & $p_{i}(\mathrm{M} 1)$ & $p_{i}(\mathrm{M} 2)$ & $p_{i}(\mathbf{M} 3)$ \\
\hline IL1 & $\mathrm{W}$ & 12.02 & 5.015 & 0.998 & 0.0165 & 0.70 & $3.39[3.26-3.69] \times 10^{-3}$ & $1.43[1.13-1.69] \times 10^{-3}$ & $7.04 \times 10^{-4}$ & 1.62 & $2.09 \times 10^{-2}$ & $1.82 \times 10^{-2}$ & $1.26 \times 10^{-2}$ \\
\hline IL2 & $\mathrm{W}$ & 6.321 & 6.893 & 0.961 & 0.0344 & 0.66 & $1.07[0.875-1.49] \times 10^{-1}$ & $5.71[3.30-7.32] \times 10^{-2}$ & $4.89 \times 10^{-2}$ & 2.23 & $8.35 \times 10^{-1}$ & $5.75 \times 10^{-1}$ & $8.78 \times 10^{-1}$ \\
\hline IL3 & $\mathrm{L}$ & 2.090 & 1.654 & 0.956 & 0.0381 & 0.70 & $5.45[3.26-9.66] \times 10^{-2}$ & $2.56[\mathrm{NA}-7.30] \times 10^{-3}$ & $3.11 \times 10^{-3}$ & 2.64 & $3.74 \times 10^{-2}$ & $2.93 \times 10^{-1}$ & $5.58 \times 10^{-2}$ \\
\hline IL4 & $\mathrm{W}$ & 17.60 & 5.141 & 0.998 & 0.0202 & 0.70 & $3.21[3.00-3.45] \times 10^{-4}$ & $1.38[1.02-1.65] \times 10^{-4}$ & $6.55 \times 10^{-5}$ & 2.46 & $2.02 \times 10^{-3}$ & $1.72 \times 10^{-3}$ & $1.18 \times 10^{-3}$ \\
\hline IL5 & $\mathrm{L}$ & 34.97 & 12.11 & 0.994 & 0.0357 & 0.57 & $1.29[1.20-1.53] \times 10^{-3}$ & $8.50[5.30-9.19] \times 10^{-4}$ & $3.65 \times 10^{-4}$ & 1.21 & $1.24 \times 10^{-2}$ & $6.93 \times 10^{-3}$ & $6.55 \times 10^{-3}$ \\
\hline IL6 & W & 11.74 & 4.118 & 0.996 & 0.0268 & 0.57 & $1.15[1.03-1.31] \times 10^{-3}$ & $4.01[2.54-5.43] \times 10^{-4}$ & $8.61 \times 10^{-5}$ & 1.35 & $5.87 \times 10^{-3}$ & $6.18 \times 10^{-3}$ & $1.55 \times 10^{-3}$ \\
\hline IL 7 & $\mathrm{~W}$ & 14.18 & 5.564 & 0.986 & 0.0530 & 0.70 & $2.43[2.02-2.92] \times 10^{-3}$ & $1.11[\mathrm{NA}-1.63] \times 10^{-3}$ & $4.45 \times 10^{-4}$ & 1.77 & $1.62 \times 10^{-2}$ & $1.31 \times 10^{-2}$ & $7.99 \times 10^{-3}$ \\
\hline IL8 & $\mathrm{W}$ & 13.58 & 5.246 & 0.994 & 0.0333 & 0.70 & $2.20[1.95-2.49] \times 10^{-3}$ & $9.61[5.10-8.60] \times 10^{-4}$ & $3.56 \times 10^{-4}$ & 2.89 & $1.41 \times 10^{-2}$ & $1.18 \times 10^{-2}$ & $6.39 \times 10^{-3}$ \\
\hline IL9 & $\mathrm{L}$ & 7.850 & 3.774 & 0.995 & 0.0096 & 0.70 & $8.32[7.70-9.05] \times 10^{-3}$ & $2.18[1.82-2.53] \times 10^{-3}$ & $1.14 \times 10^{-3}$ & 2.85 & $3.19 \times 10^{-2}$ & $4.47 \times 10^{-2}$ & $2.05 \times 10^{-2}$ \\
\hline IL10 & $\mathrm{W}$ & 7.272 & 2.692 & 0.995 & 0.0163 & 0.70 & $1.45[1.31-1.61] \times 10^{-3}$ & $2.90[1.90-3.93] \times 10^{-4}$ & $8.93 \times 10^{-5}$ & 1.68 & $4.24 \times 10^{-3}$ & $7.79 \times 10^{-3}$ & $1.60 \times 10^{-3}$ \\
\hline IL11 & $\mathrm{W}$ & 8.810 & 3.848 & 0.999 & 0.0119 & 0.57 & $4.12[2.24-5.75] \times 10^{-3}$ & $1.34[1.07-1.66] \times 10^{-3}$ & $4.39 \times 10^{-4}$ & 1.19 & $1.96 \times 10^{-2}$ & $2.21 \times 10^{-2}$ & $7.88 \times 10^{-3}$ \\
\hline M1 & $\mathrm{W}$ & 3.963 & 2.682 & 0.999 & 0.0083 & 0.66 & $2.43[2.30-2.57] \times 10^{-2}$ & $4.82[3.95-5.70] \times 10^{-3}$ & $1.88 \times 10^{-3}$ & 2.38 & & & \\
\hline M2 & $\mathrm{W}$ & 5.112 & 3.251 & 0.981 & 0.0390 & 0.66 & $2.06[1.67-2.52] \times 10^{-2}$ & $5.44[1.22-9.16] \times 10^{-3}$ & $2.11 \times 10^{-3}$ & 2.64 & & & \\
\hline M3 & $\mathrm{W}$ & 3.039 & 2.405 & 0.993 & 0.0181 & 0.66 & $3.83[3.37-4.38] \times 10^{-2}$ & $6.31[3.65-9.17] \times 10^{-3}$ & $1.93 \times 10^{-3}$ & 2.53 & & & \\
\hline
\end{tabular}

$\mathrm{M} 1, \mathrm{M} 2$, and $\mathrm{M} 3$ are mixtures of ILs in the ratios of their $\mathrm{EC}_{50}, \mathrm{EC}_{10}$, and NOEC values to their total mixture concentrations respectively, $F$ is function, $\mathrm{W}$ is Weibull function, $\mathrm{L}$ is Logit function, $\alpha$ is location parameter, $\beta$ is slope parameter, $R^{2}$ is coefficient of determination, RMSE is root mean square error, $f$ is geometric dilution factor, all the units of $\mathrm{EC}_{50}, \mathrm{EC}_{10}$, and $\mathrm{NOEC}$ are mol/L, $\mathrm{EC}_{50}$ is $50 \%$-effect concentration, $\mathrm{EC}_{10}$ is $10 \%$-effect concentration, the number in the brackets is $95 \%$ confidence interval, NA is confidence interval not available, NOEC is no observed effect concentration, $t$ is Student $t$-statistic, $p_{i}$ is concentration (mol/L) proportion of components. 
Figure 1. Concentration-response curves for the inhibitory effects of individual ionic liquids on luciferase luminescence. Hollow circle: blank control; solid circle: data observed; solid line: model fit; dash line: confidence interval.
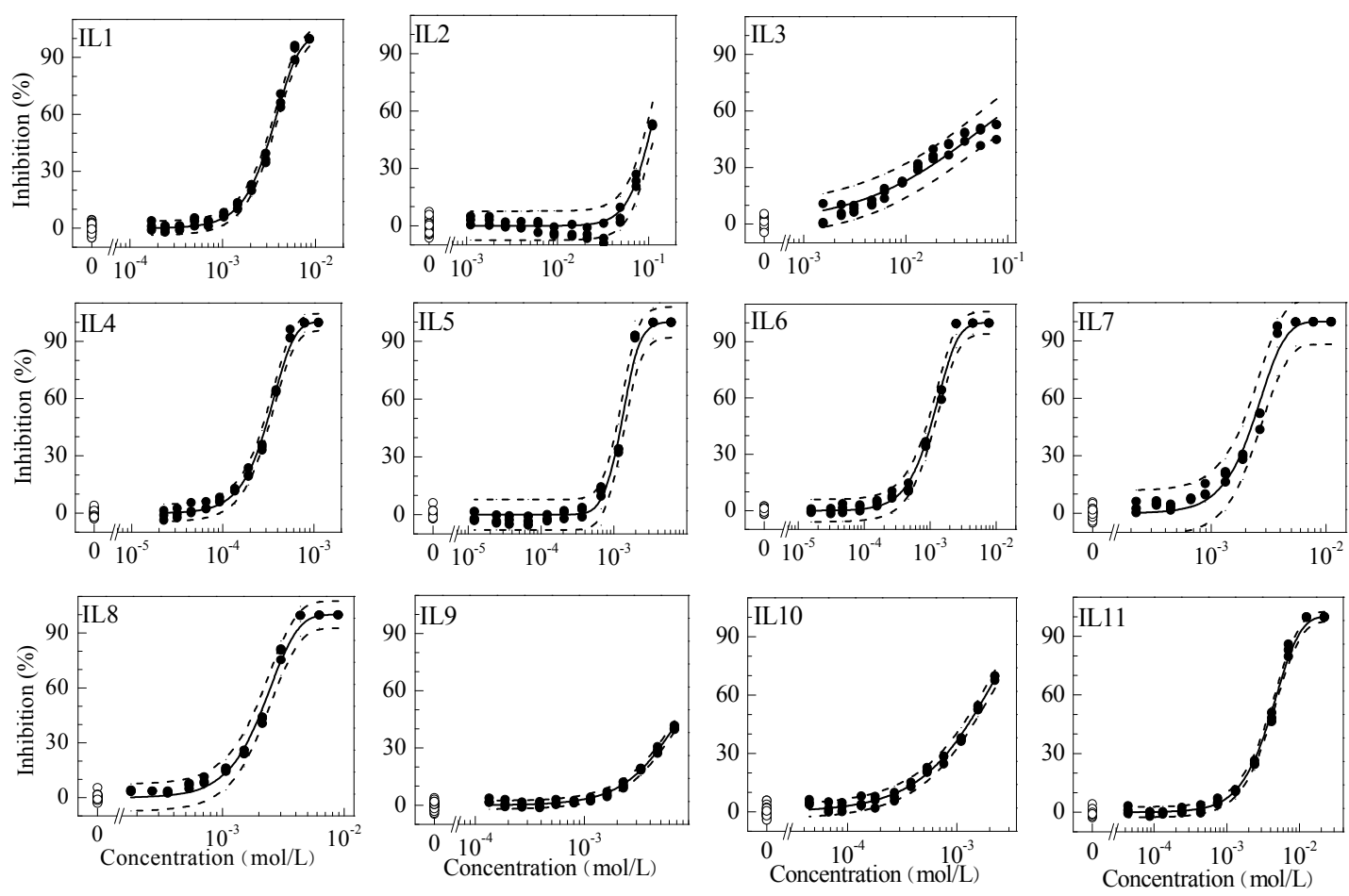

\subsection{Mixture Toxicity}

The CRCs of M1, M2 and M3 mixtures of ILs in the ratios of their $\mathrm{EC}_{50}, \mathrm{EC}_{10}$, and NOEC values respectively on luciferase can be effectively described by the Weibull model (Figure $2 \mathrm{~A}-\mathrm{C}$ ). In all cases, the $R^{2}$ s were greater than 0.98 and the RMSEs less than 0.04 . According to Table 1 , it is of interest to observe a monotonically increasing relationship between the concentration ratio of IL2 and the observed mixture toxicity $\left(\mathrm{EC}_{50}\right)$. Meanwhile, IL2 is the component having the least toxicity and the most concentration ratio in the mixtures. Figure 2D-F show the comparison of effect residual ratio (ERR) of the TSP, CA, and IA models at different effect levels for M1, M2 and M3 mixtures. The ERR of $95 \%$ confidence interval (CI) for each mixture was also given. It is possible to compare the TSP, CA, and IA models' evaluations of mixture toxicity. As a result, the toxicities of M1 can be effectively predicted by TSP, but were overestimated by CA and underestimated by IA. The toxicities of M2 can be basically predicted by TSP and CA, but were underestimated by IA. The toxicities of M3 can be predicted by TSP and CA in a certain concentration range, but were underestimated by IA. As the exposure concentration increased, the CRCs of these mixtures deviated from CA and approached IA, which could indicate that the mechanisms of action (MoAs) of ILs in the mixtures can shift with dose. For M1-M3 mixtures, CA predicted a higher effect than IA. The same situation can be found in other studies [2,3]. For this case, CA is more conservative than IA from the viewpoint of risk assessment. CA has been proved to be a more generalized approach for the effect prediction of mixtures, especially with non-monotonic CRCs [23] and with effects unable to normalize to $100 \%$ [30,31]. For mixtures consisting of different types of chemicals and deviating from additive models, we suggested that besides CA and IA, TSP should be used side by side if possible. 
Figure 2. (A-C) Concentration-response curves (CRCs) for the inhibitory effects of ionic liquid (IL) mixtures of M1 (A), M2 (B), and M3 (C) on luciferase luminescence. (D-F) Comparison of effect residual ratio (ERR) of CA, IA, and TSP models at different effect levels for mixtures of M1 (D), M2 (E), and M3 (F). (G-I) Comparison of the effects on luciferase luminescence of mixtures with those of individual ILs which concentration correspond to $\mathrm{EC}_{50} / 11(\mathbf{G}), \mathrm{EC}_{10}(\mathbf{H})$, and $\operatorname{NOEC}(\mathbf{I})$ respectively. $(\mathbf{J}-\mathbf{L})$ Comparison of the CRCs predicted by CA, IA, and TSP for three virtual mixtures of V1 (J), V2 (K), and V3 (L).
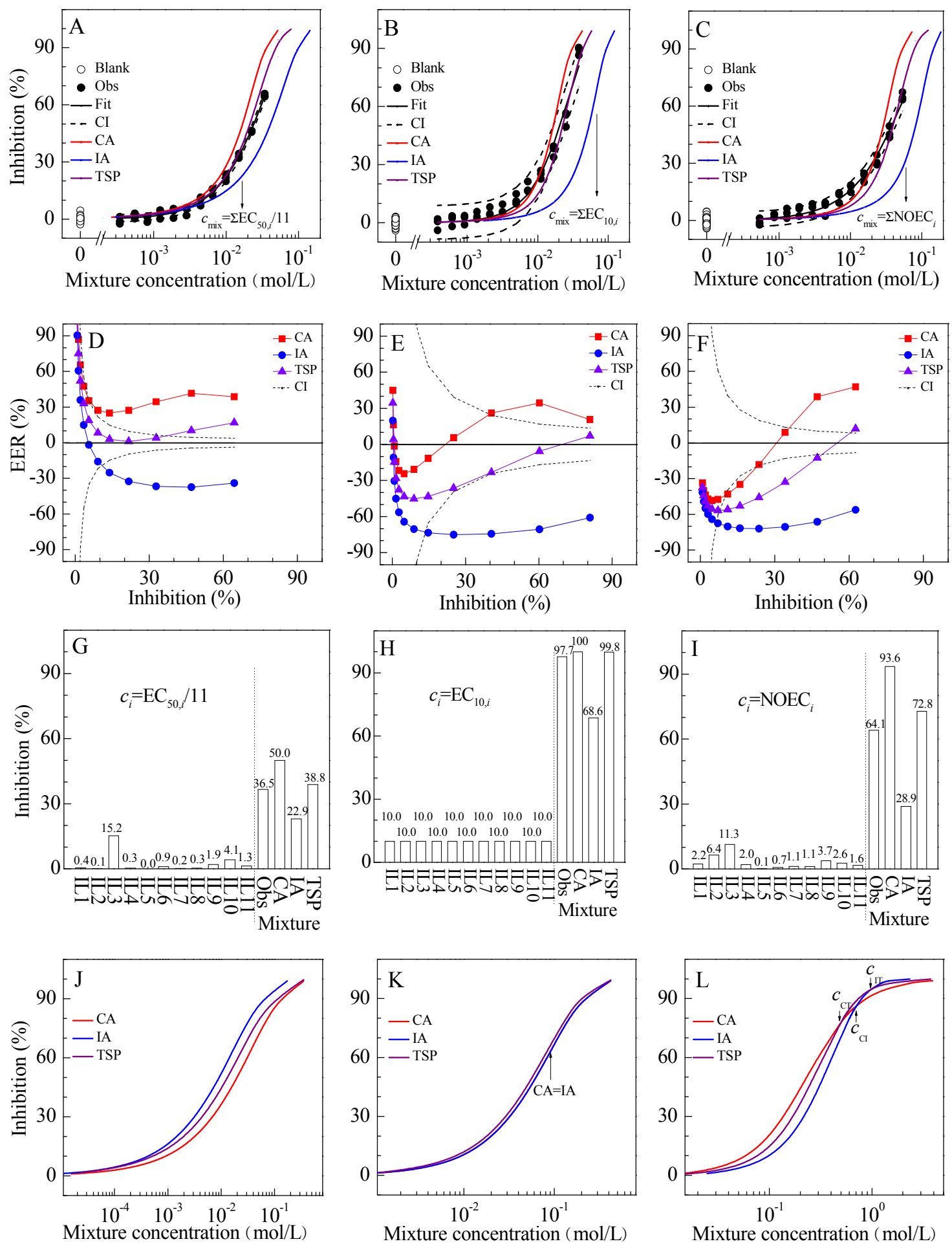
To use the TSP method effectively, it should be ensured that the concentrations and CRC models of mixture components are known, the inverse functions of component CRC models are available, and the effects can be normalized to $0-100 \%$ range. Unfortunately, the MoAs of chemicals are unknown in most cases, and sometimes one chemical may have several MoAs [32]. So it is unrealistic to determine the specific MoAs for various components. There may be certain degree of subjectivity in the TSP grouping. In this case, it is necessary to select at least one mixture for verifying the effectiveness of the grouping scheme. It has been demonstrated that the TSP according to molecular similarity grouping gives accurate prediction for the effects of M1-M3 mixtures in our study. Additionally, in the absence of knowledge on the MoAs of the components, a quantitative structure-activity relationship (QSAR) based TSP model based on structural similarity analysis might be applied to predict mixture toxicity effectively [33].

Focusing on the selected points (marked by arrows in Figure 2A-C) of the CRCs of M1, M2 and M3 mixtures, the mixtures of ILs mixed at low concentrations were found to produce significant effects (Figure 2G-I). These effects of mixtures were more effectively predicted by TSP, but were overestimated by CA and underestimated by IA. In Figure 2I, the mixture of ILs mixed at NOEC could cause significant effect of $64.1 \%$ that was nearly twice the sum (33.0\%) of component effects. Similar results can be found in the report [34]. This result emphasized the unsuitability of NOEC as an approximation of no effect concentration (NEC) especially in the context of mixtures. In Figure $2 \mathrm{H}$, the mixture of ILs mixed at $\mathrm{EC}_{10}$ could cause a significant effect of $97.7 \%$. This result indicated that $\mathrm{EC}_{10}$ as an alternative to NOEC was not necessarily conservative in the case of combined exposures. In Figure $2 \mathrm{G}$, the mixture of ILs mixed at $1 / 11$ of individual $\mathrm{EC}_{50}$ produced $36.5 \%$ effect. For the assessment factors in U.S. Food Quality Protection Act of 1996 [35], a 10-fold reduction in exposure was accounted for differences between humans and animals, a further 10-fold reduction was accounted for human-to-human variability, and another 10-fold reduction was added for protecting infants and children. In the absence of low-effect concentrations such as $\mathrm{NOEC}$ and $\mathrm{EC}_{10}$, the $\mathrm{EC}_{50}$ divided by the assessment factors may be considered for evaluating low-concentration and combined exposures. Additionally, CA predicted an effect of $50 \%$ in Figure $2 \mathrm{G}$, which proved the hypothesis that a mixture of $n$-component mixed at concentrations of $E C_{x, i} / n$ will theoretically cause an effect of $x \%$ based on CA [36].

\subsection{Location Relationship among CA, IA, and TSP Curves}

Mathematical proof indicates that CA-predicted effect will be greater than, equal to, or less than IA-predicted effect when Weibull $\beta$ values of all components are greater than, equal to, or less than 2.3 (i.e., $\ln 10$ ) [37]. In the present study, CA curves are all above IA curves for M1-M3 mixtures (i.e., CA $>$ IA), which can be attributed to Weibull $\beta$ values of the ILs being greater than 2.3 except for that of IL3 being 1.34. In this case, TSP curves are always located between the CA and IA curves. Similar results can be found in other studies [4,5].

For other cases, we designed three virtual mixtures in $\mathrm{EC}_{50}$ ratios named as V1, V2, and V3. V1 included the four components $(\mathrm{C} 1-\mathrm{C} 4)$ with Weibull parameters $(\alpha, \beta)$ as $\mathrm{C} 1(2.0,1.8), \mathrm{C} 2(2.6,1.4)$ in one group, and $\mathrm{C} 3(2.3,1.6), \mathrm{C} 4(2.9,1.2)$ in the other group. V2 included the four components (C5-C8) with Weibull parameters $(\alpha, \beta)$ as $\mathrm{C} 5(2.0,2.3)$, C6 $(2.6,2.3)$ in one group, and $\mathrm{C} 7(2.3,2.3)$, $\mathrm{C} 8(2.9,2.3)$ in the other group. V3 included the four components $(\mathrm{C} 9-\mathrm{C} 12)$ with Logit parameters $(\alpha$, 
$\beta$ ) as $\mathrm{C} 9(2.0,3.8), \mathrm{C} 10(2.6,3.8)$ in one group, and $\mathrm{C} 11(2.3,3.8), \mathrm{C} 12(2.9,3.8)$ in the other group. For V1, the order of mixture effects was IA $>$ TSP $>$ CA, and TSP curve was located between the CA and IA curves (Figure 2J). Interestingly, the order of mixture effects was TSP $>\mathrm{CA}=\mathrm{IA}$ for V2, and TSP curve was above CA and IA curves (Figure 2K). For V3, there are three intersections between $\mathrm{CA}$, IA and TSP curves at the concentrations of $c_{\mathrm{CT}}$ (CA and TSP intersecting concentration), $c_{\mathrm{CI}}$ (CA and IA intersecting concentration), and $c_{\mathrm{IT}}$ (IA and TSP intersecting concentration) with the order of $c_{\mathrm{CT}}<c_{\mathrm{CI}}<c_{\mathrm{IT}}$; TSP curve was located between the CA and IA curves in the range of concentration smaller than $c_{\mathrm{CT}}$ and greater than $c_{\mathrm{IT}}$; TSP curve was above CA and IA curves in the range of concentration greater than $c_{\mathrm{CT}}$ and smaller than $c_{\mathrm{IT}}$ (Figure 2L). Based on the results of experimental verification and numerical simulation mentioned above, we proposed a hypothesis that TSP curve will be located between the CA and IA curves when there is no overlapping or intersecting between CA and IA curves (i.e., $\mathrm{CA} \neq \mathrm{IA}$ ). This hypothesis is worthy of further experimental investigation and mathematical proof.

\section{Experimental Section}

\subsection{Chemicals}

The IL components included seven compounds from Acros (Geel, Belgium), namely 1-butyl-3methylimidazolium tetrafluoroborate (IL1, purity 99.2\%, CAS 174501-65-6), 1-butylpyridinium bromide (IL2, purity 98\%, CAS 874-80-6), 1-butyl-2,3-dimethylimidazolium chloride (IL3, purity 99.3\%, CAS 98892-75-2), 1-ethyl-3-methylimidazolium tetrafluoroborate (IL4, purity 99\%, CAS 143314-16-3), 1-benzyl-3-methylimidazolium tetrafluoroborate (IL5, purity 97\%, CAS 500996-04-3), 1-hexylpyridinium tetrafluoroborate (IL6, purity 97.5\%, CAS 474368-70-2), and 1-butyl-2,3-dimethylimidazolium tetrafluoroborate (IL7, purity 99\%, CAS 402846-78-0); three from Merck (Darmstadt, Germany), 1-butylpyridinium tetrafluoroborate (IL8, purity 99.2\%, CAS 203389-28-0), 1-hexylpyridinium bis(trifluoromethylsulfonyl)imide (IL9, purity 98\%, CAS 460983-97-5) and 1-hexyl-3methylimidazolium bis(trifluoromethylsulfonyl)imide (IL10, purity $\geq 98 \%$, CAS 382150-50-7); and one from Strem (Newburyport, MA, USA), 1-butyl-3-methylimidazolium octylsulfate (IL11, purity 98\%, CAS 445473-58-5). The chemical structures of these ILs are shown in Figure 3.

The stock solutions of these IL were prepared by dissolving them in Milli-Q water and they were stored in the dark at $4{ }^{\circ} \mathrm{C}$. The stock solutions of IL mixtures were prepared by mixing the stock solutions of individual ILs according to their assigned concentration ratios. The chemicals used in the luciferase luminescence include adenosine-5'-triphosphate (ATP-Na $\mathrm{Na}_{2} \geqslant 98.0 \%$ purity) from Sigma-Aldrich (St. Louis, MO, USA), the QuantiLum recombinant luciferase (cloned from North American firefly Photinus pyralis, Catalog \#E1701, > 95\% purity) and endotoxin-free D-luciferin (Catalog \#E6551, $\geq 98.5 \%$ purity) from Promega (Madison, WI, USA), and the glycylglycine buffer (pH 7.8, consisting of $50 \mathrm{mmol} / \mathrm{L}$ glycylglycine, $1 \mathrm{mmol} / \mathrm{L} \mathrm{MgSO}_{4}, 0.5 \mathrm{mmol} / \mathrm{L}$ EDTA, and $10 \mathrm{mmol} / \mathrm{L}$ DTT) [38]. Luciferase, luciferin, and ATP were separately stored in the glycylglycine buffer. 
Figure 3. Chemical structure of the test ionic liquids.

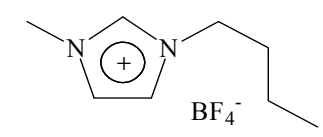

IL1

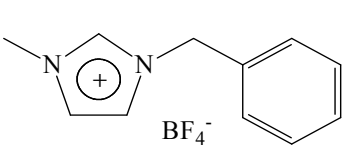

IL5

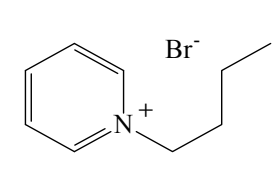

IL2

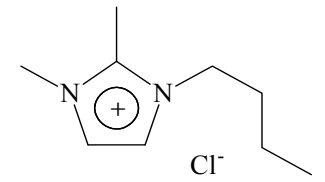

IL3

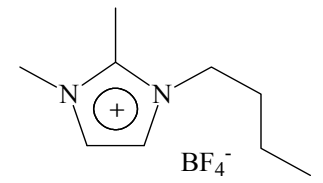

IL7

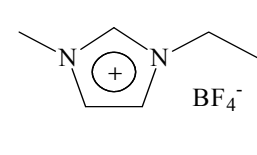

IL4

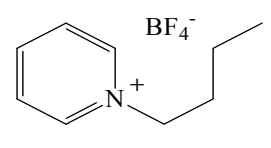

IL8

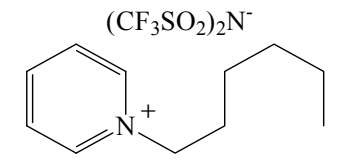

IL9

IL6

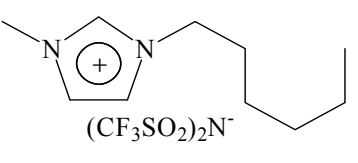

IL10

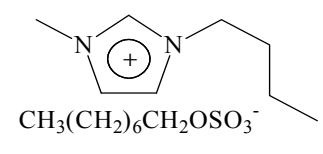

IL11

\subsection{Luciferase Toxicity Test}

The toxicities of single ILs and their mixtures were expressed as percentage inhibition of the cell-free luciferase luminescence system. According to the microplate toxicity analysis (MTA) developed in our previous study [39], the relative light units (RLUs) of the luciferase luminescence exposed to single ILs and their mixtures were determined on SpectraMax M5 reader (Molecular Devices Inc., San Francisco, CA, USA) with a 96-well white flat bottom microplate. IL chemicals and their mixtures with 12 concentration series in triplicates and 12 controls were arranged in a microplate as follows: $100 \mu \mathrm{L}$ water was added to 12 wells in the first row as blank controls, $100 \mu \mathrm{L}$ of the solutions of IL chemicals and their mixtures with 12 gradient concentrations according to a geometric dilution factors $(f)$ were added to 12 column wells from the second to the fourth row. Then, $50 \mu \mathrm{L}$ ATP of $5.5 \times 10^{-4} \mathrm{~mol} / \mathrm{L}, 50 \mu \mathrm{L}$ luciferin of $6.5 \times 10^{-5} \mathrm{~mol} / \mathrm{L}$ and $50 \mu \mathrm{L}$ luciferase of $3.3 \times 10^{-7} \mathrm{~mol} / \mathrm{L}$ were added into each test well to reach the final test volume of $250 \mu \mathrm{L}$ [40]. The test of each chemical and mixture was repeated in three microplates.

The RLUs in the microplate wells were determined after $15 \mathrm{~min}$ of exposure at $25^{\circ} \mathrm{C}$. The effect ( $E$ of $x \%$ ) of individual IL chemicals and their mixtures was calculated as Equation (1). The CRCs were fitted by Logit (L) or Weibull (W) function [3] using least squares method. The analytical formulas for the function $(F)$ and the inverse function $\left(F^{-1}\right)$ of Logit were given as Equations (2) and (3), and that of Weibull were given as Equations (4) and (5). The goodness of fit of statistical models was evaluated by coefficient of determination $\left(R^{2}\right)$ and root mean square error (RMSE). As a quantitative measure of the uncertainty, the observation-based $95 \%$ confidence interval (CI) was determined [41].

$$
\begin{gathered}
E=x \%=\left(1-L / L_{0}\right) \times 100 \% \\
E=1 /\left(1+\exp \left(-\alpha-\beta \times \log _{10}(c)\right)\right) \\
c=10^{((\ln (E /(1-E))-\alpha) / \beta)} \\
E=1-\exp \left(-\exp \left(\alpha+\beta \times \log _{10}(c)\right)\right) \\
c=10^{((\ln (-\ln (1-E))-\alpha) / \beta)}
\end{gathered}
$$


where $L_{0}$ is an average of RLUs of 12 controls, $L$ is an average of RLUs of 3 treatments, $E$ is the effect, $c$ is IL concentration, $\alpha$ is location parameter, and $\beta$ is slope parameter.

To conduct the low-concentration assessment, the no observed effect concentration (NOEC) of chemicals were determined by using Dunnett test [42]. NOEC is the highest test concentration that does not statistically significantly deviate from the control. To test the significance of this difference between treatments and control, a Student $t$-statistic was calculated according to one control in triplicates (three microplates) and 12 treatments in triplicates (three microplates). In our study, the critical value of $t$ was 2.98 for the 0.05 significance level $(a), 26$ degrees of freedom ( $d f$ ), and 12 treatments $(p)$. Then, NOEC is the highest test concentration with $t$ value less than 2.98 .

\subsection{Experimental Design and Toxicity Prediction of Mixtures}

We designed three IL mixtures named as M1, M2, and M3, where various ILs were in the ratios of their individual $\mathrm{EC}_{50, i}, \mathrm{EC}_{10, i}$, and $\mathrm{NOEC}_{i}$ to the total concentration $\left(\sum \mathrm{EC}_{50, i}, \sum \mathrm{EC}_{10, i}\right.$, and $\left.\sum \mathrm{NOEC}_{i}\right)$ of the mixtures, respectively. The mixture effects were predicted by models of CA [Equation (6)], IA [Equation (7)] [3], and TSP [4]. For TSP, since the specific MoAs of the 11 ILs on luciferase were unknown, we assigned the seven imidazolium ILs into one group and the four pyridinium ILs into the other group according to their molecular similarity. Then, the mixture effect of ILs in the same group was predicted by CA using Equation (6) in the first stage, and the overall mixture effect of the two group ILs was predicted by IA using Equation (7) in the second stage.

ERR method had been demonstrated to be an effective way for comparing the deviation of CA and IA from observation [43]. The ERR [Equation (8)] was defined as a ratio of the difference between the effect $(E)$ predicted by a reference model at a certain concentration and that observed to the observed effect. To effectively evaluate the predictability of the CA, IA, and TSP models in our study, ERR was used for the quantitative comparison of model prediction errors at different effect levels:

$$
\begin{gathered}
\sum_{i=1}^{n} \frac{c_{i}}{F_{i}^{-1}\left(E_{\mathrm{mix}, \mathrm{CA}}\right)}=1 \\
E_{\mathrm{mix}, \mathrm{IA}}=1-\prod_{i=1}^{n}\left(1-E_{i}\right) \\
\mathrm{ERR}=\frac{E_{\mathrm{Prd}}-E_{\mathrm{Obs}}}{E_{\mathrm{Obs}}} \times 100 \%
\end{gathered}
$$

where $n$ is the number of mixture components, $c_{i}$ is the concentration of the $i$ th component in the mixture, $F_{i}^{-1}$ is the inverse of function describing the CRC of the $i$ th component, $E_{\text {mix,CA }}$ is mixture effect predicted by CA, $E_{\text {mix,IA }}$ is mixture effect predicted by IA, $E_{i}$ is the effect of the $i$ th component in a mixture, $E_{\mathrm{Prd}}$ is the effect predicted by a reference model, and $E_{\mathrm{Obs}}$ is the effect observed.

\section{Conclusions}

We have studied the combined effects of seven imidazolium ILs and four pyridinium ILs on luciferase. Overall, the combined effects can be more effectively predicted by the two-stage prediction (TSP) method than the concentration addition and independent action models. This supports the use of 
the TSP method as a default approach for predicting the combined effect of different types of chemicals. It was demonstrated that even low concentrations (NOEC or $\mathrm{EC}_{10}$ ) of chemicals may lead to a significant overall effect when acting simultaneously. Therefore, the combined effects of pollutant mixtures should be taken into account in environmental risk assessment.

\section{Acknowledgements}

The authors are especially thankful to the Key Science and Technology Projects of Hainan Province (ZDXM20130043) and the National Natural Science Foundation of China (21177097) for their financial support.

\section{Author Contributions}

H.-L.G. and S.-S.L. conceived and designed the experiments. H.-L.G., B.-X.S., and X.-W.Z. performed the experiments. H.-L.G. wrote the paper and contributed to the data analysis and interpretation. All authors read and approved the final manuscript.

\section{Conflicts of Interest}

The authors declare no conflict of interest.

\section{References}

1. Suk, W.A.; Olden, K.; Yang, R.S.H. Chemical mixtures research: Significance and future perspectives. Environ. Health Perspect. 2002, 110, 891-892.

2. Backhaus, T.; Scholze, M.; Grimme, L.H. The single substance and mixture toxicity of quinolones to the bioluminescent bacterium Vibrio fischeri. Aquat. Toxicol. 2000, 49, 49-61.

3. Faust, M.; Altenburger, R.; Backhaus, T.; Blanck, H.; Boedeker, W.; Gramatica, P.; Hamer, V.; Scholze, M.; Vighi, M.; Grimme, L.H. Joint algal toxicity of 16 dissimilarly acting chemicals is predictable by the concept of independent action. Aquat. Toxicol. 2003, 63, 43-63.

4. Junghans, M. Studies on Combination Effects of Environmentally Relevant Toxicants. Ph.D. Dissertation, University of Bremen, Bremen, Germany, 2004; pp. 78-106.

5. Ra, J.S.; Lee, B.C.; Chang, N.I.; Kim, S.D. Estimating the combined toxicity by two-step prediction model on the complicated chemical mixtures from wastewater treatment plant effluents. Environ. Toxicol. Chem. 2006, 25, 2107-2113.

6. Wang, Z.; Chen, J.W.; Huang, L.P.; Wang, Y.; Cai, X.Y.; Qiao, X.L.; Dong, Y.Y. Integrated fuzzy concentration addition-independent action (IFCA-IA) model outperforms two-stage prediction (TSP) for predicting mixture toxicity. Chemosphere 2009, 74, 735-740.

7. Pham, T.P.T.; Cho, C.W.; Yun, Y.S. Environmental fate and toxicity of ionic liquids: A review. Water Res. 2010, 44, 352-372.

8. Zhang, J.; Liu, S.S.; Liu, H.L. Effect of ionic liquid on the toxicity of pesticide to Vibrio-qinghaiensis sp.-Q67. J. Hazard. Mater. 2009, 170, 920-927. 
9. Freire, M.G.; Neves, C.M.S.S.; Carvalho, P.J.; Gardas, R.L.; Fernandes, A.M.; Marrucho, I.M.; Santos, L.M.; Coutinho, J.A.P. Mutual solubilities of water and hydrophobic ionic liquids. $J$. Phys. Chem. B 2007, 111, 13082-13089.

10. Luo, Y.R.; Wang, S.H.; Yun, M.X.; Li, X.Y.; Wang, J.J.; Sun, Z.J. The toxic effects of ionic liquids on the activities of acetylcholinesterase and cellulase in earthworms. Chemosphere 2009, 77, 313-318.

11. Ranke, J.; Molter, K.; Stock, F.; Bottin-Weber, U.; Poczobutt, J.; Hoffmann, J.; Ondruschka, B.; Filser, J.; Jastorff, B. Biological effects of imidazolium ionic liquids with varying chain lengths in acute Vibrio fischeri and WST-1 cell viability assays. Ecotoxicol. Environ. Safe. 2004, 58, 396-404.

12. Cho, C.W.; Jeon, Y.C.; Pham, T.P.T.; Vijayaraghavan, K.; Yun, Y.S. The ecotoxicity of ionic liquids and traditional organic solvents on microalga Selenastrum capricornutum. Ecotoxicol. Environ. Safe. 2008, 71, 166-171.

13. Kumar, R.A.; Papaiconomou, N.; Lee, J.M.; Salminen, J.; Clark, D.S.; Prausnitz, J.M. In vitro cytotoxicities of ionic liquids: Effect of cation rings, functional groups, and anions. Environ. Toxicol. 2009, 24, 388-395.

14. Matzke, M.; Stolte, S.; Arning, U.; Uebers, U.; Filser, J. Imidazolium based ionic liquids in soils: Effects of the side chain length on wheat (Triticum aestivum) and cress (Lepidium sativum) as affected by different clays and organic matter. Green Chem. 2008, 10, 584-591.

15. Costello, D.M.; Brown, L.M.; Lamberti, G.A. Acute toxic effects of ionic liquids on zebra mussel (Dreissena polymorpha) survival and feeding. Green Chem. 2009, 11, 548-553.

16. Pretti, C.; Chiappe, C.; Baldetti, I.; Brunini, S.; Monni, G.; Intorre, L. Acute toxicity of ionic liquids for three freshwater organisms: Pseudokirchneriella subcapitata, Daphnia magna and Danio rerio. Ecotoxicol. Environ. Safe. 2009, 72, 1170-1176.

17. Niedermeyer, H.; Hallett, J.P.; Villar-Garcia, I.J.; Hunt, P.A.; Welton, T. Mixtures of ionic liquids. Chem. Soc. Rev. 2012, 41, 7780-7802.

18. Matzke, M.; Stolte, S.; Boschen, A.; Filser, J. Mixture effects and predictability of combination effects of imidazolium based ionic liquids as well as imidazolium based ionic liquids and cadmium on terrestrial plants (Triticum aestivum) and limnic green algae (Scenedesmus vacuolatus). Green Chem. 2008, 10, 784-792.

19. Zhang, J.; Liu, S.S.; Dou, R.N.; Liu, H.L.; Zhang, J. Evaluation on the toxicity of ionic liquid mixture with antagonism and synergism to Vibrio qinghaiensis sp.-Q67. Chemosphere 2011, 82, 1024-1029.

20. Zhang, J.; Liu, S.S.; Yu, Z.Y.; Liu, H.L. Significant contributions of ionic liquids containing tetrafluoroborate and trifluoromethanesulfonate to antagonisms and synergisms in multi-component mixtures. J. Hazard. Mater. 2012, 209-210, 158-163.

21. Rodionova, N.S.; Petushkov, V.N. Effect of different salts and detergents on luciferin-luciferase luminescence of the enchytraeid Fridericia heliota. J. Photochem. Photobiol. B 2006, 83, $123-128$.

22. Kamaya, H.; Ueda, I.; Eyring, H. Combined effects of dissociable and undissociable local anesthetics upon ATP-induced firefly bioluminescence. Proc. Natl. Acad. Sci. USA 1976, 73, 1868-1871. 
23. Ge, H.L.; Liu, S.S.; Zhu, X.W.; Liu, H.L.; Wang, L.J. Predicting hormetic effects of ionic liquid mixtures on luciferase activity using the concentration addition model. Environ. Sci. Technol. 2011, 45, 1623-1629.

24. Ge, H.L.; Liu, S.S.; Su, B.X.; Qin, L.T. Predicting synergistic toxicity of heavy metals and ionic liquids on photobacterium Q67. J. Hazard. Mater. 2014, 268, 77-83.

25. Stolte, S.; Arning, J.; Bottin-Weber, U.; Matzke, M.; Stock, F.; Thiele, K.; Uerdingen, M.; Welz-Biermann, U.; Jastorff, B.; Ranke, J. Anion effects on the cytotoxicity of ionic liquids. Green Chem. 2006, 8, 621-629.

26. Egorova, K.S.; Ananikov, V.P. Toxicity of ionic liquids: Eco(cyto)activity as complicated, but unavoidable parameter for task-specific optimization. ChemSusChem 2014, 7, 336-360.

27. Fox, D. NECS, NOECS and the EC $\mathrm{E}_{\mathrm{x}}$. Australas. J. Ecotoxicol. 2008, 14, 7-9.

28. Warne, M.S.J.; van Dam, R. NOEC and LOEC data should no longer be generated or used. Australas. J. Ecotoxicol. 2008, 14, 1-5.

29. Van der Hoeven, N.; Noppert, F.; Leopold, A. How to measure no effect. Part I: Towards a new measure of chronic toxicity in ecotoxicology. Introduction and workshop results. Environmetrics 1997, 8, 241-248.

30. Howard, G.J.; Schlezinger, J.J.; Hahn, M.E.; Webster, T.F. Generalized concentration addition predicts joint effects of aryl hydrocarbon receptor agonists with partial agonists and competitive antagonists. Environ. Health Perspect. 2010, 118, 666-672.

31. Liu, S.S.; Liu, L.; Chen, F. Application of the concentration addition model in the assessment of chemical mixture toxicity (in Chinese). Acta Chim. Sin. 2013, 71, 1335-1340.

32. Syberg, K.; Elleby, A.; Pedersen, H.; Cedergreen, N.; Forbes, V.E. Mixture toxicity of three toxicants with similar and dissimilar modes of action to Daphnia magna. Ecotoxicol. Environ. Safe. 2008, 69, 428-436.

33. Kim, J.; Kim, S.; Schaumann, G.E. Development of QSAR-based two-stage prediction model for estimating mixture toxicity. SAR QSAR Environ. Res. 2013, 24, 841-861.

34. Silva, E.; Rajapakse, N.; Kortenkamp, A. Something from "nothing"-Eight weak estrogenic chemicals combined at concentrations below NOECs produce significant mixture effects. Environ. Sci. Technol. 2002, 36, 1751-1756.

35. EPA. Food Quality Protection Act of 1996. Public Law 104-170, 1996.

36. Könemann, H. Structure-activity relationships and additivity in fish toxicities of environmental pollutants. Ecotoxicol. Environ. Safe. 1980, 4, 415-421.

37. Boedeker, W.; Drescher, K.; Altenburger, R.; Faust, M.; Grimme, L. Combined effects of toxicants: The need and soundness of assessment approaches in ecotoxicology. Sci. Total Environ. 1993, 134, 931-939.

38. Hu, J.Z. A Study on the Properties and Applications of Firefly Luciferase (in Chinese). Master Thesis, East China Normal University, Shanghai, China, 2007; pp. 28-29.

39. Zhang, Y.H.; Liu, S.S.; Song, X.Q.; Ge, H.L. Prediction for the mixture toxicity of six organophosphorus pesticides to the luminescent bacterium Q67. Ecotoxicol. Environ. Safe. 2008, 71, 880-888.

40. Ge, H.L.; Liu, S.S.; Chen, F.; Luo, J.H.; Lv, D.Z.; Su, B.X. Microplate luminometry for toxicity bioassay of chemicals on luciferase (in Chinese). Spectrosc. Spect. Anal. 2013, 33, 2766-2770. 
41. Zhu, X.W.; Liu, S.S.; Ge, H.L.; Liu, Y. Comparison between two confidence intervals of dose-response relationships (in Chinese). China Environ. Sci. 2009, 29, 113-117.

42. Dunnett, C.W. New tables for multiple comparisons with a control. Biometrics 1964, 20, 482-491.

43. Wang, L.J.; Liu, S.S.; Zhang, J.; Li, W.Y. A new effect residual ratio (ERR) method for the validation of the concentration addition and independent action models. Environ. Sci. Pollut. Res. 2010, 17, 1080-1089.

Sample Availability: Not available.

(C) 2014 by the authors; licensee MDPI, Basel, Switzerland. This article is an open access article distributed under the terms and conditions of the Creative Commons Attribution license (http://creativecommons.org/licenses/by/3.0/). 